Document downloaded from:

http://hdl.handle.net/10251/64612

This paper must be cited as:

Martínez Pinzón, G.; López Sánchez, J.; Gómez Barquero, D.; Cardona Marcet, N. (2015). Optimization of the Digital Terrestrial Television Transmission Mode of DVB-T2 in Colombia. IEEE Latin America Transactions. 13(7):2144-2151. doi:10.1109/TLA.2015.7273770.

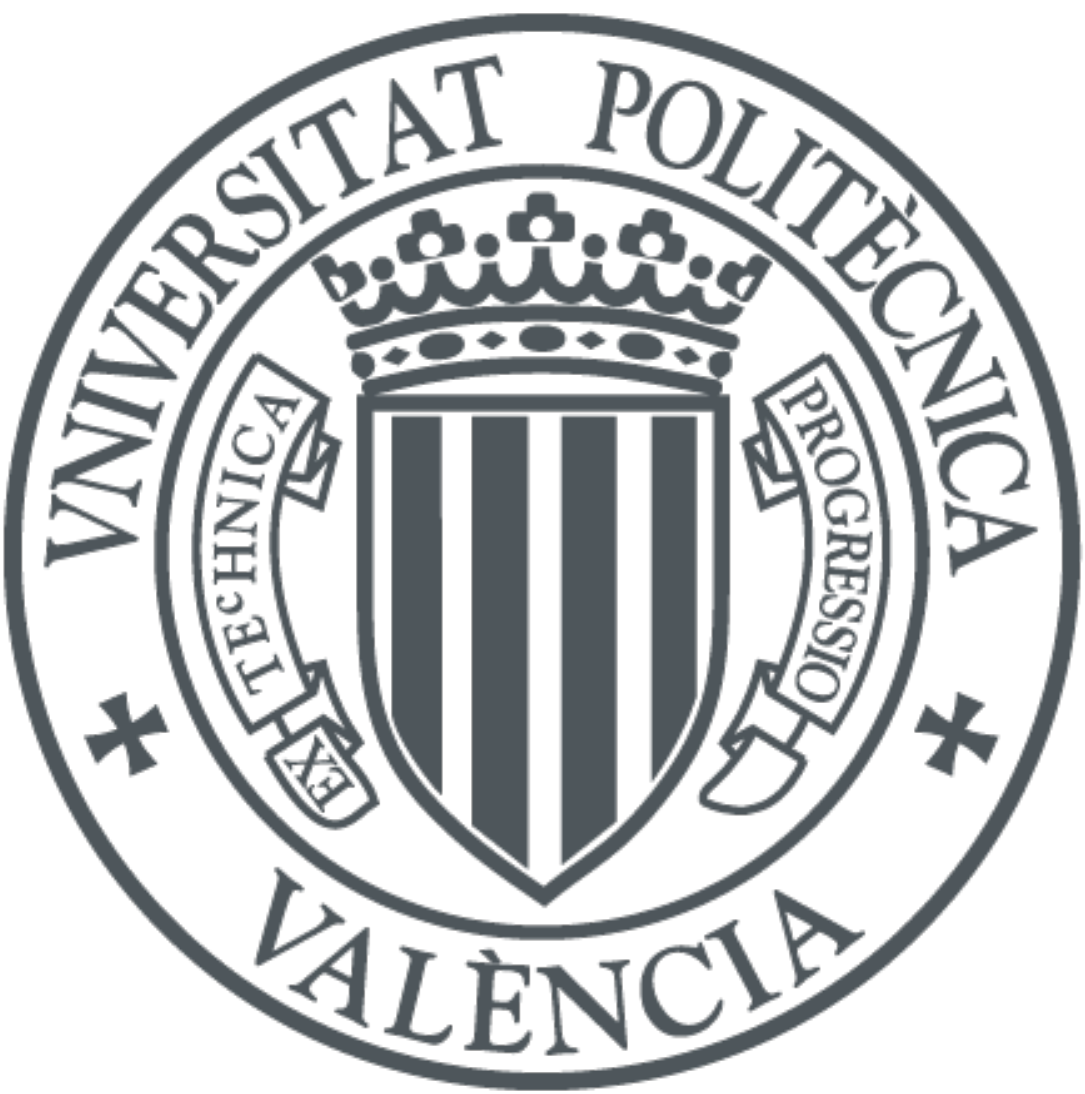

The final publication is available at

http://dx.doi.org/10.1109/TLA.2015.7273770

Copyright Institute of Electrical and Electronics Engineers (IEEE)

Additional Information 


\title{
Optimization of the Digital Terrestrial Television Transmission Mode of DVB-T2 in Colombia
}

\author{
G. Martínez-Pinzón, J. López-Sánchez, D. Gomez-Barquero and N. Cardona
}

\begin{abstract}
In December 2011, Colombia updated its national Digital Terrestrial Television (DTT) standard from DVB-T to DVB-T2, the second generation of the DVB (Digital Video Broadcasting) project. DVB-T2 is the current state-of-the art DTT system in the world, and it brings very significant improvements in terms of capacity, robustness and flexibility compared with its predecessor. The case of Colombia is very special because it was the first country to deploy DVB-T2 with $6 \mathrm{MHz}$ channelization and because the DVB-T2 networks were deployed from scratch without any constraint imposed by existing DVB-T infrastructure. This paper analyzes potential DVB-T2 transmission modes to optimize existing DTT network designs of Colombia, originally made for DVB-T, analyzing the trade-offs between capacity, coverage level and Single Frequency Network (SFN) size. Network planning results has been performed with a professional DTT networkplanning tool based on the three first deployment phases of the DTT network of the public national TV broadcaster RTVC. Results obtained fully justify the decision of adopting DVB-T2. Compared with the initial DVB-T design, DVB-T2 can increase the national covered population up to $7.3 \%$ or offer $70.2 \%$ more of capacity transmission, or increase the SFN size up to $135 \%$ keeping the total power transmission and the geographic distribution of the transmitters.
\end{abstract}

Keywords - Digital Terrestrial Television, Network Planning, Single Frequency Network, DVB-T, DVB-T2.

\section{INTRODUCCIÓN}

$\mathrm{D}$ VB-T2 (Digital Video Broadcasting - Terrestrial 2nd generation) es el estándar de segunda generación para la radiodifusión de Televisión Digital Terrestre (TDT) más avanzado en el mundo, ya que ofrece velocidades de transmisión de hasta $50 \%$ superior respecto a su antecesor DVB-T (Digital Video Broadcasting - Terrestrial 1st generation), para un mismo ancho de banda [1]. DVB-T2 brinda la posibilidad de transmitir diferentes tipos de servicios (TV en calidad estándar (SDTV), TV en alta definición (HDTV), TV tridimensional (3DTV)) en un mismo múltiplex (frecuencia), con diferentes parámetros de transmisión.

Si comparamos el estándar de segunda generación frente a su antecesor, DVB-T2 incorpora los últimos avances tecnológicos en modulación y codificación del canal. Asimismo, ofrece un alto grado de eficiencia, flexibilidad y robustez. Esta robustez de la señal se puede traducir en un

G. Martínez, Universitat Politècnica de València (UPV), Valencia, Spain, gemarpin@iteam.upv.es

J. Sánchez, Universitat Politècnica de València (UPV), Valencia, Spain, jailosan@iteam.upv.es

D. Barquero, Universitat Politècnica de València (UPV), Valencia, Spain, dagobar@iteam.upv.es

N. Cardona, Universitat Politècnica de València (UPV), Valencia, Spain ,ncardona@iteam.upv.es aumento de la cobertura o un ahorro de energía si se establece el mismo porcentaje de cobertura objetivo [1].

Colombia adoptó en 2008 el estándar DVB-T para la provisión de la TDT. No obstante, teniendo en cuenta que la red del operador Radio Televisión Pública de Colombia (RTVC) aún no estaba desplegada, se decidió en el año 2011 actualizar el estándar de TDT a DVB-T2, debido principalmente a las ventajas técnicas que ofrece y por ende la oportunidad de nuevos modelos de negocio [2].

RTVC había diseñado la red en topología MFN (MultiFrequency Network) utilizando dos frecuencias y la posibilidad de asignar una tercera frecuencia para transmisores fronterizos que puedan generar interferencia con otros países. El objetivo de cobertura poblacional aproximadamente era del 65\% para diciembre del 2014 cubriendo las principales ciudades del país y alcanzar hasta el 92,2\% para el año 2019, fecha en la que se proyecta el apagón analógico [3]. Sin embargo, RTVC ha tenido que adaptar su diseño inicial de red por la adopción de DVB-T2. Su estrategia inicial era mantener la topología de red ofreciendo la misma cobertura poblacional pero maximizando la capacidad de transmisión. Esta solución ya había sido adoptada en países como Reino Unido y Suecia pero con la diferencia que sus redes ya habían sido desplegadas en DVB-T. Sin embargo, para cambiar el diseño de red de RTVC nuevas estrategias pueden plantearse para aprovechar las ventajas de DVB-T2, considerando que su red aún no está desplegada.

DVB-T2 implementa tamaños grandes de FFT e intervalos de guarda permitiendo desplegar redes SFN (Single Frequency Network) de gran tamaño y por lo tanto aumentar el área de cobertura. En consecuencia, podría implementarse una red SFN nacional reduciendo el número de frecuencias necesarias para cubrir el país. Así, Colombia podría ser el primer país en desplegar la red DVB-T2 más grande del mundo en topología SFN con canalización $6 \mathrm{MHz}$ respecto a la canalización $8 \mathrm{MHz}$ utilizada en Europa. La canalización $6 \mathrm{MHz}$ permite una separación máxima entre transmisores de $212 \mathrm{~km}$ en vez de $179 \mathrm{~km}$ a $8 \mathrm{MHz}$. Teniendo en cuenta estas consideraciones proponemos un estudio exhaustivo para maximizar los beneficios que ofrece esta tecnología.

Este artículo analiza potenciales modos de transmisión DVB-T2 en términos de ganancias en capacidad de transmisión, cobertura y tamaño de red SFN, para actualizar los diseños iniciales de red DVB-T en Colombia. Adicionalmente, se propone una metodología para optimización de la red de TDT que maximice el uso del espectro. La optimización de la red es llevada a cabo en tres pasos. Primero, se evaluarán diferentes configuraciones de modos de transmisión T2 y se escoge el modo de transmisión 
de red que ofrece mejores prestaciones en cuanto a capacidad, cobertura y tamaño de red SFN. Segundo, se modifica los retardos artificiales de la señal de salida de los transmisores, con el fin de minimizar las auto-inferencias de la red SFN. De igual forma, se realiza un ajuste manual de dichos retardos con el objetivo de que las zonas que aún continuaban interferidas, sean zonas geográficas donde no interesa ofrecer cobertura. Finalmente, se podrían desplegar transmisores de baja potencia conocidos como gap-fillers, en aquellas zonas donde aún existen interferencias para eliminarlas.

Los resultados de planificación de red han sido obtenidos usando la herramienta de software profesional ICS Telecom. El escenario de estudio está compuesto por 38 transmisores de alta potencia correspondientes a las tres primeras fases de RTVC [4] [5].

El artículo está estructurado de la siguiente forma: En la Sección II, una comparativa técnica de DVB-T2 respecto a su antecesor DVB-T. En la Sección III, se explica la metodología llevada a cabo para la estimación de cobertura y la optimización de red SFN. En la Sección IV, se presentan los resultados de este artículo. Finalmente, las conclusiones y recomendaciones en la Sección V.

\section{VENTAJAS DEL ESTÁNDAR DVB-T2 RESPECTO A SU ANTECESOR DVB-T}

DVB-T2 ofrece un amplio rango de modos de transmisión convirtiéndolo en un estándar muy flexible. DVB-T2 incluye importantes innovaciones en los parámetros de configuración técnicos: mecanismos nuevos de corrección de errores (FEC), altos órdenes de modulación (256QAM), tamaños nuevos de FFT (16K y 32K) permitiendo utilizar el modo extendido, fracciones nuevas de intervalos de guarda (GI) y diferentes opciones de patrones de portadoras piloto (PP) optimizados en función del GI. Adicionalmente, se añaden nuevas funcionalidades, tales como, tuberías de capa física (PLPs), constelaciones rotadas, entrelazado temporal, mecanismos de reducción de relación de potencia pico a nivel medio (PAPR), tramas de extensión futura (FEFs), y el perfil para servicios móviles conocido como T2-Lite [6]. La TABLA I resume sus principales diferencias.

Los PLPs permiten la provisión de diferentes tipos de servicios en un mismo multiplex de forma más flexible, al poder utilizar una modulación y codificación diferente para cada servicio. Por ejemplo, los servicios en movilidad requieren modos de transmisión más robustos frente a ruido e interferencias que los servicios fijos. Las constelaciones rotadas consiguen obtener una señal transmitida más robusta que es útil para canales con desvanecimientos selectivos en tiempo y frecuencia. Esto se implementa agregando un giro a todos los puntos de la constelación. El entrelazado temporal se basa en transmitir los datos en diferentes instantes de tiempo, de manera que si se produce una perturbación de la señal OFDM, esta afecte a bits que no son consecutivos, con el objetivo de detectar y corregir los errores con mayor facilidad. La técnica de PAPR es utilizada para la reducción en los picos de potencia del amplificador de RF, permitiendo un menor
TABLA I. DIFERENCIAS ENTRE DVB-T VS. DVB-T2.

\begin{tabular}{|c|c|c|}
\hline PARAMETRO & DVB-T & DVB-T2 \\
\hline $\begin{array}{l}\text { FEC y Tasa de } \\
\text { Codificación }\end{array}$ & $\begin{array}{c}\mathrm{CC}+\mathrm{RS} \\
1 / 2,2 / 3,3 / 4, \\
5 / 6,7 / 8\end{array}$ & $\begin{array}{c}\mathrm{LDPC}+\mathrm{BCH} \\
1 / 2,3 / 5,2 / 3,3 / 4,4 / 5,5 / 6\end{array}$ \\
\hline Modulaciones & $\begin{array}{l}\text { QPSK, 16QAM, } \\
\text { 64QAM }\end{array}$ & $\begin{array}{l}\text { QPSK, 16QAM, 64QAM, } \\
\text { 256QAM (opcional rotado) }\end{array}$ \\
\hline Tamaño FFT & $2 \mathrm{~K}, 8 \mathrm{~K}$ & $1 \mathrm{~K}, 2 \mathrm{~K}, 4 \mathrm{~K}, 8 \mathrm{~K}, 16 \mathrm{~K}, 32 \mathrm{~K}$ \\
\hline $\begin{array}{l}\text { Intervalo de } \\
\text { Guarda }\end{array}$ & $\begin{array}{c}1 / 4,1 / 8,1 / 16 \\
1 / 32\end{array}$ & $\begin{array}{c}1 / 4,19 / 128,1 / 8,19 / 256, \\
1 / 16,1 / 32,1 / 128\end{array}$ \\
\hline Overhead por PPs & $8 \%$ del total & $1 \%, 2 \%, 4 \%, 8 \%$ del total \\
\hline $\begin{array}{l}\text { Capacidad } \\
\text { Máxima@ @ } 9 \mathrm{Mzz}\end{array}$ & $23.8 \mathrm{Mbps}$ & 37.0 Mbps \\
\hline
\end{tabular}

consumo de energía y por ende una disminución en el costo de operación de una red [7]. El perfil T2-Lite permite transmitir nuevos servicios para recepción en movilidad en un modo de configuración totalmente independiente sobre las tramas DVB-T2 base.

\section{A. Ganancia en Cobertura}

Los códigos FEC se encuentran en el límite de las tecnologías de codificación. La información se protege por dos nuevos códigos FEC introducidos: el LPDC (Low-Density Parity-Check) y el BCH (Bose-Chaudhuri-Hochquenghem). El rendimiento conjunto de estos dos mecanismos FEC está a tan solo $1 \mathrm{~dB}$ por debajo del límite de Shannon en canales gaussianos. Comparado con los Códigos Convolucional (CC) y Reed-Salomon (RS) utilizados en DVB-T, se obtienen ganancias desde $2.3 \mathrm{~dB}$ de Relación Portadora a Ruido (CNR) para servicios de baja tasas de transmisión hasta $3.2 \mathrm{~dB}$ para servicios de altas tasas transmisión en canales estacionarios [7]. Dependiendo de la topología de red y las condiciones geográficas del escenario, esto puede equivaler al doble de área cubierta.

Las constelaciones rotadas también proporcionan una robustez adicional a la señal, especialmente para órdenes bajos de modulación y tasas altas de codificación. La ganancia depende del canal, oscilan de 0.5 a $2 \mathrm{~dB}$, pero para órdenes altos de modulación (64QAM, 256QAM) la ganancia es casi despreciable. La ganancia SFN ofrecida por la técnica de Alamouti al utilizar diversidad en transmisión MISO (Multiple Input Single Output) puede alcanzar hasta $2.5 \mathrm{~dB}$, dependiendo de los tiempos de retardo entre las señales y de los niveles de potencia de cada una de ellas [8].

\section{B. Ganancia en Capacidad}

DVB-T2 puede alcanzar hasta 50\% más en capacidad respecto a su antecesor DVB-T [8]. Altos órdenes de modulación (256QAM) poseen una mayor eficiencia espectral y son decodificados con umbrales similares de CNR a los requeridos en DVB-T para modulaciones más robustas. Esto es debido principalmente a la eficiencia de los códigos FEC.

Por otra parte, los tamaños de FFT $16 \mathrm{~K}$ y 32K, tienen largas duraciones de símbolo OFDM que significa menor porcentaje de la capacidad de transmisión dedicada a protección, para un mismo intervalo de guarda, si utilizáramos una FFT de 8K del estándar DVB-T. 
TABLA II.

MÁXIMA DISTANCIA SFN (KM) ENTRE TRANSMISORES A 6 MHZ.

\begin{tabular}{cccccccc}
\hline \hline Tamaño & \multicolumn{7}{c}{ FFT } \\
\cline { 2 - 8 } & $\mathbf{1 / 1 2 8}$ & $\mathbf{1 / 3 2}$ & $\mathbf{1 / 1 6}$ & $\mathbf{1 9 / 2 5 6}$ & $\mathbf{1 / 8}$ & $\mathbf{1 9 / 1 2 8}$ & $\mathbf{1 / 4}$ \\
\hline $\mathbf{3 2 K}$ & 11,2 & 44,8 & 89,6 & 106,4 & 179,2 & 212,8 & N/A \\
$\mathbf{1 6 K}$ & 5,6 & 22,4 & 44,8 & 53,2 & 89,6 & 106,4 & 179,2 \\
$\mathbf{8 K}$ & 2,8 & 11,2 & 22,4 & 26,6 & 44,8 & 53,2 & 89,6 \\
$\mathbf{4 K}$ & N/A & 5,6 & 11,2 & N/A & 22,4 & N/A & 44,8 \\
$\mathbf{2 K}$ & N/A & 2,8 & 5,6 & N/A & 11,2 & N/A & 22,4 \\
$\mathbf{1 K}$ & N/A & N/A & 2,8 & N/A & 5,6 & N/A & 11,2 \\
\hline
\end{tabular}

Asimismo, los tamaños más altos de FFT permiten la implementación de modos extendidos, estos modos extendidos permite aumentar el número de portadoras, con una ganancia de aproximadamente del $2 \%$ en la capacidad de transmisión [8].

DVB-T2 define 8 patrones de portadoras piloto con el fin de minimizar el overhead (entre 1\% al 8,3\%), en función del tipo de recepción al que este orientado el servicio, en comparación con DVB-T que solo permite un único patrón de portadoras, el cual representa un 8,3 \% de overhead [9].

\section{Ganancia SFN}

Los parámetros que definen el tamaño de una red SFN son el tiempo del intervalo de guarda del sistema $(T g)$, que depende de la combinación del tamaño de la FFT y la fracción del intervalo de guarda. Las señales que llegan al receptor con un retardo menor que la duración del intervalo de guarda, contribuye constructivamente en recepción, de lo contrario presentarán un efecto de interferencia entre símbolos (ISI) [10]. La duración máxima del intervalo de guarda en DVB-T con canalización $6 \mathrm{MHz}$ es $298 \mu$ s, para el modo FFT 8K GI 1/4 [11], lo que corresponde a una distancia entre transmisores de $89 \mathrm{~km}$. DVB-T2 permite alcanzar una separación máxima entre transmisores de $212 \mathrm{~km}$ para el modo (FFT 32K, GI 19/128, Tg 709 s) con canalización 6 MHz. La TABLA II resume las máximas distancias permitidas en un sistema DVB-T2 con canalización $6 \mathrm{MHz}$ en función del tamaño de FFT y del intervalo de guarda.

\section{METODOLOGÍA PARA LA ESTIMACIÓN DE COBERTURA}

\section{A. Escenario de partida}

El escenario de estudio es el diseño de red DVB-T del operador RTVC que consta de 38 transmisores de alta potencia entre 200W a 5kW. Los parámetros técnicos de configuración de red (potencia de emisión de las estaciones, configuración sistema radiante, ubicación de las estaciones, etc.) están basados en información real proporcionada por el operador [4] [5]. Las simulaciones han sido hechas en la banda UHF (Ultra High Frequency), concretamente se usaron las frecuencias $485 \mathrm{MHz}$ y 527 (canales 16 y 23) para los estudios en topología MFN y $485 \mathrm{MHz}$ (canal 16) para estudios en topología SFN.
La cartografía usada en las simulaciones está compuesta por: un mapa digital del terreno con resolución planimétrica de 30m, una capa de clutter dividida en 12 categorías (urbano, suburbano, pastos, cultivos bajos, bosque, selva, desierto, etc.) y una capa de la división política de los municipios de Colombia (1123) con la población al año 2014 [12]. La metodología empleada para los estudios hechos fue validada por la Agencia Nacional del Espectro (ANE).

\section{A. Estimación área de cobertura}

Un punto es considerado cubierto, sí la intensidad de campo eléctrica total útil $\left(E_{\min }\right)$ y la mínima relación portadora a ruido más interferencia $\left(C N I R_{\min }\right)$ superan los umbrales mínimos requeridos por el sistema (para determinado modo de recepción) [13].

\section{B. Mínimas Relación Portadora a Ruido $\left(C N R_{\text {min }}\right) e$ Intensidad de Campo Eléctrico $\left(E_{\text {min }}\right)$}

En [14] se obtienen los umbrales mínimos de CNR en dB requeridos para una correcta decodificación en el receptor, teniendo en cuenta el canal de propagación en función del tipo de recepción. Estos valores de CNR incluyen un factor de corrección que permite modelar el comportamiento de los receptores reales. El cálculo de la intensidad de campo mínima ha sido obtenido aplicando la ecuación (1) [14]:

$$
E_{\text {min }}=P_{N}+C N R_{\text {min }}-A_{a}+L_{f}+C_{L}+145.8
$$

Donde $P_{N}$ es la potencia de ruido térmico, $A_{a}$ representa la apertura efectiva de la antena, $L_{f}$ son las perdidas en los alimentadores y $C_{L}$ representa un factor de corrección de emplazamiento, que depende de la probabilidad de localización (70\% cobertura aceptable para recepción fija y 95\% cobertura buena para recepción portable), con una desviación estándar de 5.5 dB.

\section{Balance del enlace}

La potencia a la entrada del receptor $P_{R x}$ puede ser estimada de acuerdo a la ecuación (2):

$$
P_{R x}=P_{T x}+G_{T x}+G_{R x}-L_{T x-R x}-L_{P}-L_{H}-L_{B}
$$

Donde $P_{T x}$ es la potencia transmitida, $G_{T x}$ es la ganancia de la antena transmisora, $G_{R x}$ representa la ganancia de la antena receptora, $L_{T x-R x}$ son las pérdidas de cables y conectores del transmisor y receptor, $L_{P}$ son las pérdidas de propagación calculadas usando el modelo ITU-R 525/526 con método de difracción Deygout 94 [15] [16]. Ha sido tenido en cuenta una ganancia de $11 \mathrm{dBd}$ para recepción fija sobre tejado y $0 \mathrm{dBd}$ para recepción portable [17]. La altura de la antena receptora es $10 \mathrm{~m}$ sobre el nivel del suelo para recepción fija y $1.5 \mathrm{~m}$ para recepción portable. $L_{H}$ es un factor de perdida por altura que se considera para recepción portable [14]. Asimismo, un valor de $11 \mathrm{~dB}$ ha sido tenido en cuenta por la pérdida que sufre la señal al atravesar un edificio $L_{B}$ en recepción indoor [18]. 


\section{Cálculo de la relación portadora a ruido más interferencia (CNIR)}

La $C N I R$ depende de la potencia de la señal útil $P_{U}$, la potencia interferente propia de la SFN $P_{I, \text { self }}$, la potencia interferente externa debido a otros sistemas de comunicación operando a la misma frecuencia $P_{I, \text { ext }}$, y la potencia de ruido $P_{N}$. Todos los parámetros son considerados lineales [19].

$$
C N I R=\frac{P_{U}}{P_{I, \text { self }}+P_{I, \text { ext }}+P_{N}}
$$

El cálculo de $P_{U}$ es derivado de la ecuación (4). La señal recibida con mayor intensidad de campo se considera como la señal principal mientras las otras señales que llegan al receptor son consideradas como ecos.

$$
P_{u}=\sum_{i \epsilon S F N} P_{r i} \times W_{i}(\tau)
$$

Dependiendo del retardo relativo $\tau$ entre los ecos y la señal principal, los retardos pueden contribuir a la señal constructivamente y/o interferentemente. Esta contribución es definida mediante una función de peso $W_{i}(\tau)$, especificada en la ecuación (5) [8].

$$
W_{i}(\tau)=\left\{\begin{array}{cl}
0, & \text { if } \tau \notin T_{I E} \\
\left(\frac{T_{U}+\tau}{T_{U}}\right)^{2}, & \text { if } \tau \in T_{I E} \& \tau<0 \\
1, & \text { if } \tau \in T_{I E} \& 0 \leq \tau \leq T_{g} \\
\left(\frac{T_{U}+T_{g}-\tau}{T_{U}}\right)^{2}, & \text { if } \tau \in T_{I E} \& \tau>T_{g}
\end{array}\right.
$$

Donde $T_{U}$ es la parte constructiva del símbolo OFDM y $T_{I E}$ es el intervalo máximo de ecualización que está definido por el límite de Nyquist, que depende del patrón de portadoras piloto [14]. El parámetro $P_{I, \text { self }}$, puede ser calculado aplicando la ecuación (6).

$$
P_{I, \text { self }}=\sum_{i \in S F N} P_{r i} \times\left[1-W_{i}(\tau)\right]
$$

\section{E. Combinación de señales OFDM}

Las estimaciones de cobertura SFN presentadas en este artículo han sido calculadas mediante una sumatoria no estadística de las señales de potencia individuales. La señal útil en una SFN, es representada por la suma de las potencias de las señales útiles. Para las señales no deseadas, los valores medios de potencias son sumados al mínimo nivel de señal útil presente (representando la contribución de ruido) [17].

\section{F. Cálculo retardo artificial en los transmisores}

Debido a los efectos de multicamino que degradan a la señal transmitida, y dependiendo de la topología de la red SFN donde transmisores pueden estar localizados a distancias mayores que la distancia permitida SFN (depende del tamaño de la FFT y la fracción del GI), algunas contribuciones de la señal transmitida pueden llegar al receptor fuera del intervalo de ecualización. En ese caso, esas contribuciones son consideradas destructivas, generando interferencias. Una solución es la introducción de un retardo artificial en la señal de salida del transmisor con la intención de que al receptor lleguen dentro del intervalo antes citado, y por lo tanto puede mitigar estas interferencias

Esta optimización en redes SFN es muy importante porque no conlleva ningún coste adicional en la infraestructura de red y logra reducir significativamente las interferencias. En redes SFN compuestas por más de 5 transmisores calcular manualmente los retardos artificiales puede resultar muy complejo debido a la dependencia entre ellos. Nuestro método de optimización de los retardos artificiales es realizado en dos pasos: En primer lugar, se obtiene los retardos artificiales $\delta$, mediante una herramienta de planificación de red profesional. El procedimiento llevado a cabo por el software es calcular en cada punto de las zonas de cobertura solapadas el tiempo de llegada de las señales al receptor. Seguidamente, en las zonas interferidas de cada transmisor, se evalúa un rango de intervalos de ecualización $[-\delta, \delta]$ que permitan minimizar dichas áreas. Asimismo se realiza el procedimiento para todos los transmisores que conformen la red hasta encontrar la mejor combinación que ofrece la menor área cubierta interferida. En segundo lugar, se realiza un ajuste manual de los retardos artificiales permitiendo desplazar las interferencias SFN a zonas geográficas con nula o baja densidad poblacional (montañas, desiertos, etc.), permitiendo asimismo maximizar la cobertura. Este paso es llevado a cabo porque la herramienta de planificación no tiene en cuenta las zonas que quedan interferidas ni la densidad poblacional dentro de estas, sino solo porcentaje de área cubierta interferida.

\section{MODOS DE TRANSMISIÓN PARA COLOMBIA}

Diferentes modos de transmisión DVB-T2 han sido evaluados y comparados con el modo DVB-T inicialmente seleccionado por RTVC, para identificar la mejor configuración de red que mejores prestaciones ofrece en términos de ganancia en cobertura, capacidad y distancia SFN.

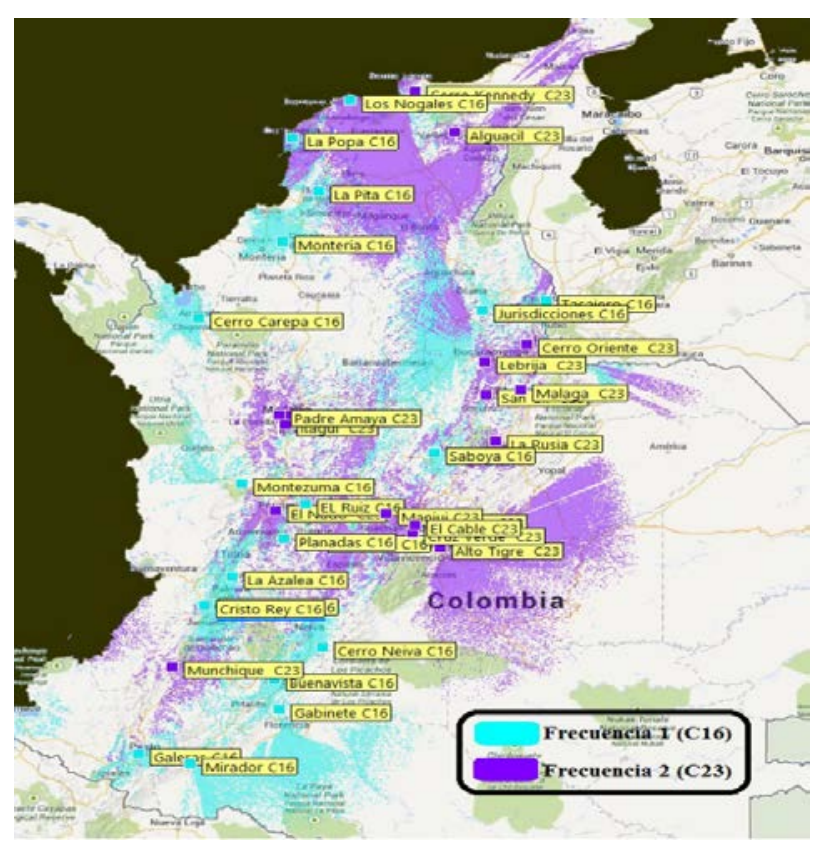

Figura 1. Huella de cobertura DVB-T para recepción fija en topología MFN (dos frecuencias) en Colombia. 
TABLA III.

MODOS DE TRANSMISIÓN DVB-T2 QUE MAXIMIZAN EL ÁREA DE COBERTURA.

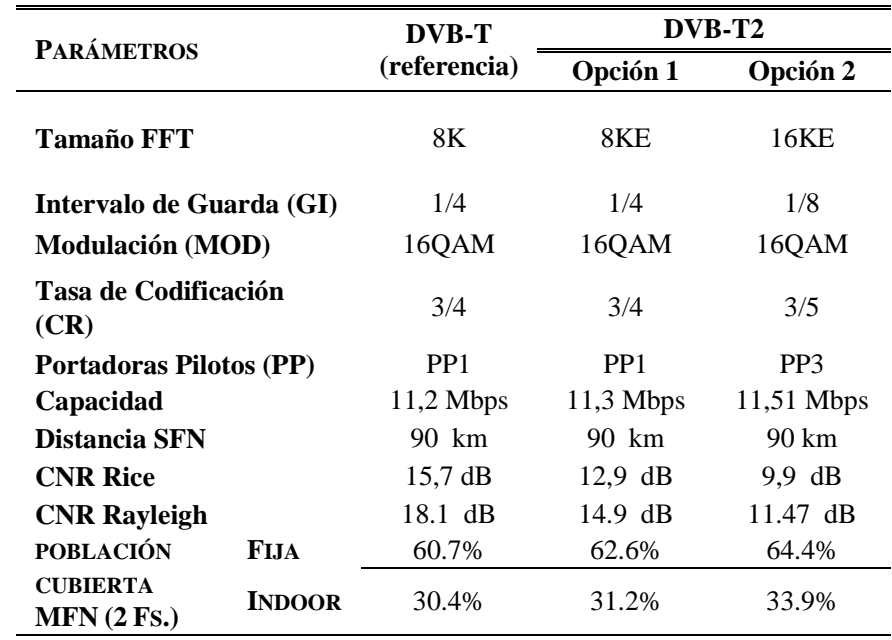

La Fig. 1 muestra las huellas de cobertura del diseño de red DVB-T basado en una combinación de topologías de red MFN (utilizando dos frecuencias) y SFNs regionales. El modo DVB-T (de ahora en adelante modo de referencia) seleccionado era: MOD 16QAM, CR 3/4, FFT 8K, GI 1/4. Este modo requiere un umbral mínimo de CNR de $15.7 \mathrm{~dB}$ para recepción fija y $17.4 \mathrm{~dB}$ para recepción portable, ofreciendo una capacidad de 11.2 Mbps y una separación entre transmisores SFN de $90 \mathrm{~km}$ (distancia máxima alcanzada por DVB-T).

\section{A. Ganancia en Cobertura}

La Tabla III presenta dos modos de transmisión DVB-T2 que ofrecen la misma capacidad de transmisión que el modo de referencia DVB-T y mantienen la distancia entre transmisores SFN de $90 \mathrm{~km}$. No obstante, T2 ofrece más robustez a la señal, pudiéndose reflejar en una reducción de CNR. Los resultados obtenidos para el modo de referencia muestran una cobertura poblacional de $60,7 \%$ para recepción fija y 30,4 \% para recepción portable en topología MFN.

El modo DVB-T2 opción 1 tiene los mismos parámetros de transmisión que el modo de referencia DVB-T, pero requiere $2.8 \mathrm{~dB}$ menos de CNR, principalmente por las mejoras introducidas en codificación FEC. Esta reducción de CNR se traduce en una ganancia de cobertura poblacional de 1,9\% y $0,8 \%$ para recepción fija y portable, respecto al diseño original en topología MFN.

El modo DVB-T2 opción 2 utiliza un tamaño más grande de FFT permitiendo reducir la fracción del GI, esta combinación permite mantener la distancia SFN entre transmisores de $90 \mathrm{~km}$. También tiene un patrón de portadoras menos denso. Con esta configuración se consigue reducir el overhead, que permite un incremento en la tasa de transmisión, pudiendo ser aprovechada con una tasa de codificación más robusta que mantenga la misma capacidad. Sin embargo, utilizar tamaños más grandes de FFT implica una penalización en recepción en movilidad. En este caso, manteniendo el orden de modulación 16QAM es posible usar

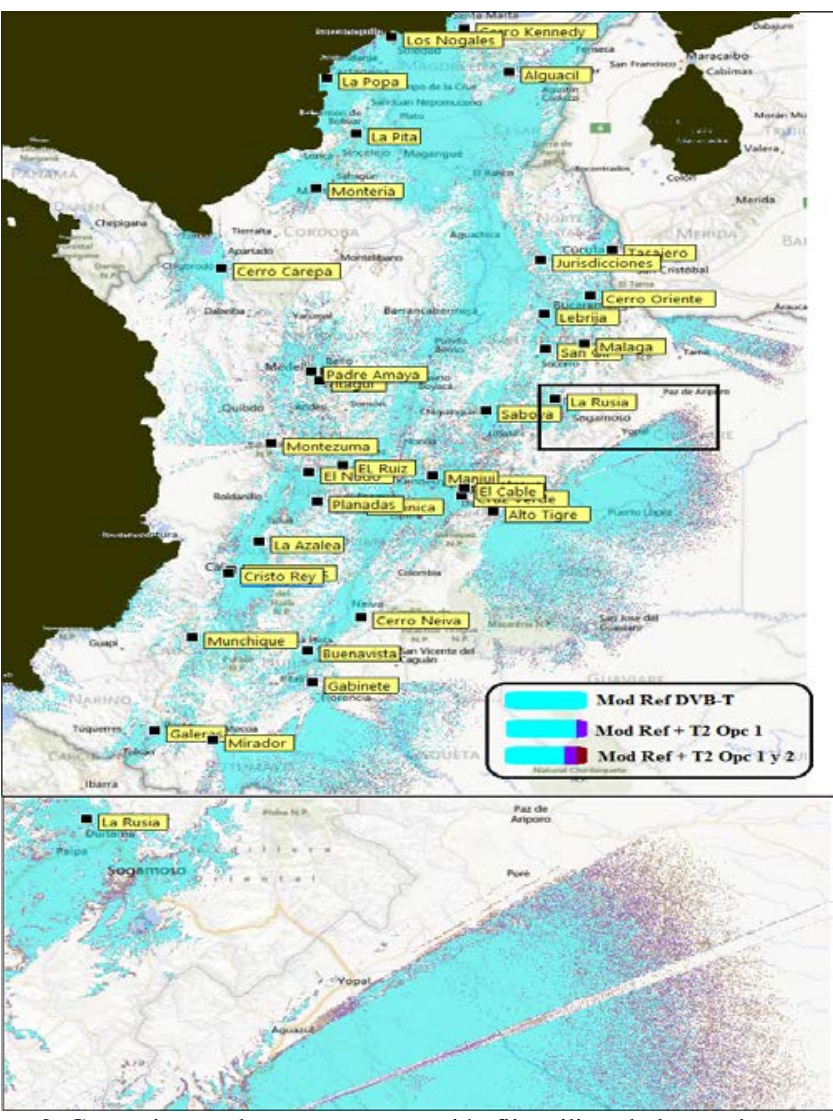

Figura 2. Ganancia en cobertura para recepción fija utilizando los modos DVB-T2 propuestos.

una tasa de codificación de 3/5, logrando una reducción de CNR de $5.8 \mathrm{~dB}$ que se traslada en un incremento de cobertura poblacional de $3.7 \%$ tanto para recepción fija y portable.

La Fig. 2 muestra la superposición de las huellas de cobertura obtenidas para el modo de referencia DVB-T y los modos DVB-T2 opción 1 y 2 donde se puede ver claramente la ganancia de cobertura obtenida por los modos propuestos.

\section{B. Ganancia en Capacidad}

La Tabla IV presenta un modo de transmisión DVB-T2 (opción 3) que requiere un nivel de CNR ligeramente mayor que el modo de referencia, pero en términos de área cubierta es prácticamente igual. Por otro lado, hay dos modos (opción 4 y 5) que necesitan aproximadamente $1 \mathrm{~dB}$ menos de CNR respecto al modo de referencia, traduciéndose en una ganancia en cobertura.

Todos los modos propuestos DVB-T2 permiten utilizar un orden de modulación más alto (64QAM), que se refleja en un incremento en la eficiencia espectral, como consecuencia de las mejoras en la codificación LDPC. Además, los tres modos propuestos tienen la misma duración temporal para el intervalo de guarda, aunque la combinación tamaño de FFT y fracción del intervalo de guarda sean diferentes. Por lo tanto, una ganancia adicional en capacidad es obtenida, debido a la reducción del overhead y la utilización de patrones de portadoras pilotos menos densos.

EL modo DVB-T2 opción 3 alcanza una ganancia en capacidad del $70 \%$ con tan solo una reducción del área de cobertura del 0,2\%. 
TABLA IV.

MODOS DE TRANSMISIÓN DVB-T2 QUE MAXIMIZAN LA CAPACIDAD DE TRANSMISIÓN.

\begin{tabular}{|c|c|c|c|c|}
\hline \multirow[b]{2}{*}{ PARÁMETROS } & \multirow{2}{*}{$\begin{array}{c}\text { DVB-T } \\
\text { (referencia) }\end{array}$} & \multicolumn{3}{|c|}{ DVB-T2 } \\
\hline & & $\begin{array}{c}\text { OPCIÓN } \\
3 \\
\end{array}$ & $\begin{array}{c}\text { OPCIÓN } \\
4 \\
\end{array}$ & $\begin{array}{c}\text { OPCIÓN } \\
5 \\
\end{array}$ \\
\hline Tamaño FFT & $8 \mathrm{~K}$ & $16 \mathrm{KE}$ & $32 \mathrm{KE}$ & $16 \mathrm{KE}$ \\
\hline $\begin{array}{l}\text { Intervalo de } \\
\text { Guarda }\end{array}$ & $1 / 4$ & $1 / 8$ & $1 / 16$ & $1 / 8$ \\
\hline Modulación & 16QAM & 64QAM & 64QAM & 64QAM \\
\hline $\begin{array}{l}\text { Tasa de } \\
\text { Codificación }\end{array}$ & $3 / 4$ & $2 / 3$ & $3 / 5$ & $3 / 5$ \\
\hline Patrón Piloto & PP1 & PP3 & PP4 & PP3 \\
\hline CNR Rice & $15,7 \mathrm{~dB}$ & $16,1 \mathrm{~dB}$ & $14,8 \mathrm{~dB}$ & $14,8 \mathrm{~dB}$ \\
\hline Distancia SFN & $90 \mathrm{~km}$ & $90 \mathrm{~km}$ & $90 \mathrm{~km}$ & $90 \mathrm{~km}$ \\
\hline Capacidad & $\begin{array}{c}\text { 11,2 } \\
\text { Mbps }\end{array}$ & $\begin{array}{c}\text { 19,2 } \\
\text { Mbps }\end{array}$ & $\begin{array}{l}18,59 \\
\text { Mbps }\end{array}$ & $\begin{array}{l}17,25 \\
\text { Mbps }\end{array}$ \\
\hline $\begin{array}{l}\text { Población } \\
\text { Cubierta } \\
\text { Recepción Fija, } \\
\text { MFN (2 Fs.) }\end{array}$ & $60,7 \%$ & $60,5 \%$ & $60.9 \%$ & $60.9 \%$ \\
\hline
\end{tabular}

Los modos opción 4 y 5 ofrecen ganancias en capacidad del $66 \%$ y 54\% respectivamente. El modo opción 4 ofrece una ganancia en capacidad significativa, pero comparado con los otros dos modos DVB-T2, tiene peores prestaciones en movilidad.

\section{A. Ganancia en Tamaño SFN}

EL tamaño SFN elegido inicialmente por RTVC era de 90 km, el máximo permitido por DVB-T. La Tabla V presenta tres modos DVB-T2 que permiten tamaños grandes de SFN y altas capacidades de transmisión respecto al modo de referencia. El nivel de CNR requerido por todos es casi igual al del modo de referencia. Asimismo, todos los modos propuestos DVB-T2 incrementan el orden de modulación a 64QAM, y tasa de codificación 3/5 con el fin de incrementar la capacidad.

El modo DVB-T2 opción 6 permite una distancia máxima entre transmisores de $212 \mathrm{~km}$, máxima distancia permitida en DVB-T2 con canalización 6 MHz. Los modos opción 7 y 8 ofrecen niveles de cobertura e interferencia similares al modo opción 6, por lo tanto una separación entre transmisores de $180 \mathrm{~km}$ es suficiente para la distribución prevista de la red primaria de RTVC considerando la orografía Colombiana. La principal ventaja que ofrece el modo opción 7 es la alta tasa de transmisión, sin embargo tamaños grandes de FFT tienen una penalización para servicios en movilidad. Por otro lado, el modo opción 8 ofrece mejores prestaciones para servicios en movilidad pero la ganancia en capacidad es solo del 20,2\%.

Si comparamos los resultados presentados en la Tabla $\mathrm{V}$ respecto a la Tabla IV, el modo DVB-T en topología MFN con dos frecuencias alcanza una cobertura poblacional sin optimizar los retardos artificiales $1,8 \%$ menor que los modos DVB-T2 (opciones 3, 4 y 5) a una sola frecuencia. Adicionalmente, se presentan resultados de cobertura e interferencia tras optimizar los retardos artificiales en los transmisores. Se consiguen reducciones significativas de áreas interferidas para los modos de transmisión que permiten menores distancias SFN. Alrededor del 1\% de la población es TABLA V.

MODOS DE TRANSMISIÓN DVB-T2 PARA MÁXIMIZAR TAMAÑO SFN. RESULTADOS PARA RECEPCIÓN FIJA SOBRE TEJADO.

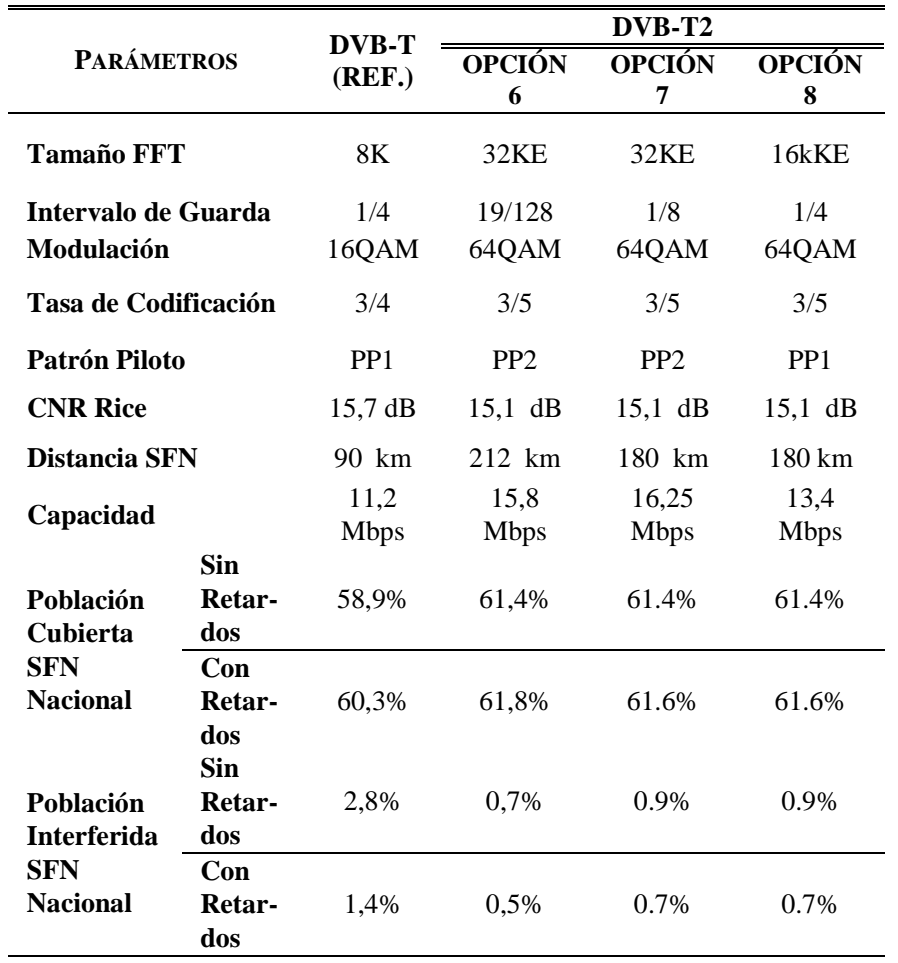

cubierta donde antes era interferida, debido al incremento del nivel de la señal deseada en aquellas zonas donde dos o más señales de diferentes transmisores son recibidas dentro del intervalo de guarda (llamada también ganancia SFN). Por último, la Fig. 3 muestra la superposición de las huellas de interferencias para el modo DVB-T frente a los modos DVBT2 propuestos, en la zona noroccidental del país. Se observa como disminuyen las zonas de interferencias a medida que la distancia entre transmisores es mayor, obteniéndose resultados similares para los modos opción 7 y 8.

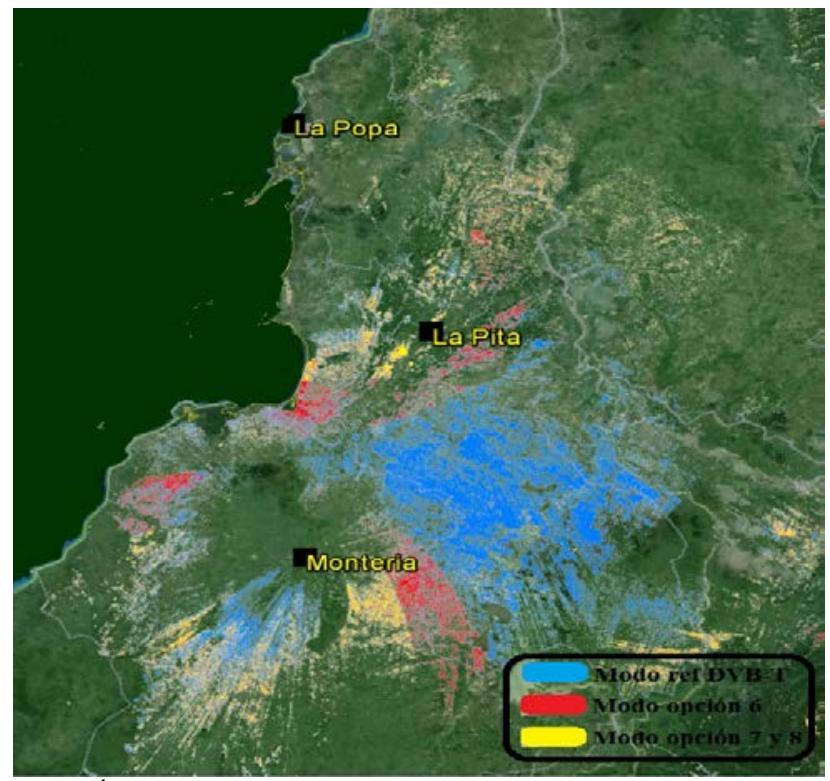

Figura 3. Áreas con auto-interferencia de la red SFN Nacional. 


\section{CONCLUSIONES Y TRABAJO FUTURO}

Colombia ha actualizado el estándar de TDT a DVB-T2, el sistema más avanzado del mundo, que permite ofrecer más capacidad, robustez y flexibilidad que cualquier otro sistema. Colombia podría ser el primer país en desplegar la red DVB-T2 más grande del mundo en topología SFN con canalización $6 \mathrm{MHz}$. Nuestras investigaciones demuestran que comparando el modo inicialmente elegido por RTVC, DVBT2 puede proporcionar una ganancia en cobertura de más de 5 $\mathrm{dB}$ ofreciendo la misma capacidad o una ganancia en capacidad de $70 \%$ para la misma área de cobertura. Igualmente, nuestros resultados demuestran que es posible desplegar una red SFN nacional cubriendo todo el país con tan solo una frecuencia en vez de dos frecuencias como lo requería el diseño inicial para DVB-T. Después de optimizar los retardos artificiales en los transmisores es posible reducir el porcentaje de población interferida a tan solo el 0,5\% de la población Colombiana, que podría ser fácilmente eliminado usando transmisores de baja potencia o gap-fillers. Esta red SFN sería la más grande del mundo, con una distancia máxima entre transmisores entre 180 y $212 \mathrm{~km}$.

Los resultados indican que $180 \mathrm{~km}$ es suficiente para la distribución geográfica de los transmisores planificados, logrando así una ganancia en capacidad del $45 \%$ y manteniendo la misma área de cobertura del diseño inicial DVB-T. La única desventaja de los modos DVB-T2 que maximizan la cobertura, capacidad o tamaño SFN es que se utilizan tamaños de FFT de 32K, obteniéndose menores prestaciones para servicios en movilidad. Más investigaciones podrían llevarse a cabo para evaluar las prestaciones en movilidad de DVB-T2 con canalización $6 \mathrm{MHz}$ en función del tamaño de la FFT y portadoras piloto. Además, la utilización de tuberías de capa física o el perfil en movilidad T2-lite, podrían proporcionar de manera eficiente servicios fijos y móviles con la misma infraestructura y el mismo canal. Otra optimización adicional de la red podría ser la transmisión de servicios nacionales y regionales.

\section{AGRADECIMIENTOS}

Este trabajo ha sido validado por la Agencia Nacional del Espectro (ANE) de Colombia.

\section{REFERENCIAS}

[1] I. Eizmendi, M. Velez, D. Gómez-Barquero, J. Morgade, V. Baena Lecuyer, M. Slimani, J. Zoellner, "DVB-T2: The Second Generation of Terrestrial Digital Video Broadcasting System", IEEE Transactions on Broadcasting, vol. 60, no. 2, pp. 258-271, June 2014.

[2] F. A. Contreras, E. Pedraza-Barrera, and D. Gómez-Barquero, "On the Transmission of Mobile Digital Terrestrial Television DVB-T2 Services in Colombia, , Communications and Computing (COLCOM) ", 2014 IEEE Colombian Conference, vol., no., pp.1,6, 4-6 June 2014.

[3] ANTV, "Modificación artículo décimo primero del acuerdo 002, Prestación del servicio público de televisión abierta radiodifundida digital terrestre TDT”, 2012.

[4] RTVC-SP-07, "Estudios previos proceso de selección pública”, 2013.

[5] RTVC-IA-02, “Estudios previos invitación abierta Fase II TDT”, 2014
[6] D. Gozálvez, D. Gómez-Barquero, I. Eizmendi, G. Berjón-Eriz, and M. Vélez, “DVB-T2 for mobile and Mobile DVB-T2 (T2-Lite),” in Next generation mobile broadcasting, 1st ed. Vol. 1, Ed. CRC press, 2013, pp. 151-184.

[7] D. Gómez-Barquero, J. López-Sánchez, G. Martínez, J. RibadeneiraRamirez, E. Garro, C. García-Pardo, M. Fuentes, and N. Cardona, "Frequency and Network Planning and Optimization of the Digital Terrestrial Television DVB-T2 Networks in Colombia", Waves, year 6, 2014.

[8] ETSI Std. EN 302 755, "Frame structure channel coding and modulation for a second generation digital terrestrial television broadcasting system (DVB-T2)”, Rev. 1.2.1, 2011.

[9] J. López-Sánchez, C. García Acero, D. Gómez-Barquero and N. Cardona, "Planning a Mobile DVB-T Network for Colombia," IEEE Latin America Transactions, Vol. 8, no. 4, Aug. 2010.

[10] R. Egli, S.Torres, L. Pradenas, J. R. Pérez and J. Basterrechea, "Maximizing Coverage of a Single Frequency Network for Digital Television Services,” IEEE Latin America Transactions, Vol. 10, no. 4, June 2012.

[11] ETSI Std. EN 300 744, "Framing structure, channel coding and modulation for digital terrestrial television”, Rev. 1.6.1, 2009.

[12] DANE, “Estimaciones y proyecciones de población 1985 - 2020”, Abril 2010.

[13] ETSI Std. TR 102 831, "Implementation guidelines for a second generation digital terrestrial television broadcasting system (DVB-T2)”, Rev. 0.10.4, 2010.

[14] EBU TECH 3348, "Frequency and Network Planning Aspects of DVB-T2”, Report, Version 3.02013.

[15] ITU-R P.525-2, “Calculation of free-space attenuation”, 1994.

[16] ITU-R P.526-13, "Propagation by diffraction”, Geneva, 2013.

[17] ITU, "DTTB Handbook Digital Terrestrial Television Broadcasting in VHF/UHF bands”, V1.01, pp. 152-155, 2012.

[18] F. A. Contreras, E. Pedraza-Barrera, and D. Gómez-Barquero, "DVB-T2 field trials results for portable indoor reception in Colombia", Proc. IEEE Latin-America Conference on Communications (LATINCOM), 2014.

[19] A. Mattson, "Single Frequency Networks in DTV," IEEE Transactions on Broadcasting, vol. 51, no. 4, pp. 413-422, December 2005.

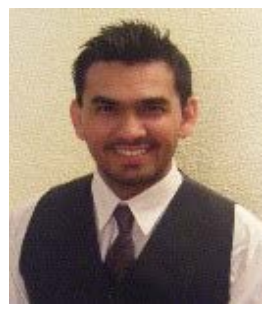

Gerardo Martínez Pinzón received a double Telecommunications Engineer degree at Technical University of Valencia, Spain and Saint Thomas Aquinas University, Colombia. In 2013, he has finished a Master in Development of Mobile Communications Systems. More than 3 years of experience in the mobile communications, digital terrestrial television and electronic security systems fields. He has developed research activities and consulting providing recommendations on multiple projects from the initial design stages to planning, optimization, coexistence and deployment. Specialized in the latest telecommunications technologies such as LTE-Advance mobile communications or DVB-T, DVB-T2 and ISDB-T digital terrestrial television (DTT) standards. His current research activities are focused on efficient spectrum management through the use of cognitive radio technologies.

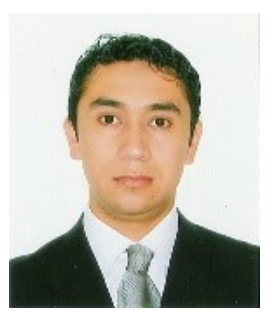

Jaime Lopez-Sánchez received Electronic Engineering degree from the Universidad Distrital Francisco José de Caldas, Bogotá, Colombia, and the Ph.D. degree in telecommunications from the Universidad Politècnica de Valencia, Spain, in 2005 and 2014, respectively. During the doctoral studies, he was a Guest Researcher at the Institute for Communications Technology, Technische Universität Braunschweig, Braunschweig, Germany, for three months. He has participated in several research and development projects such as FURIA, AV-MOV, and Redes Híbridas in the study and research of digital video broadcasting (DVB) technologies. He was the recipient of the first prize in the research contest "Valencia idea 2010". His current research interests include new technical solutions in the evolution of DVB standards for provisioning of advanced multimedia services in digital television networks. 


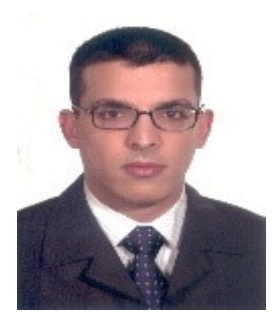

David Gómez-Barquero received the double M.Sc. degrees in telecommunications engineering from the Universitat Politècnica de Valencia (UPV), Spain, and the University of Gavle, Sweden, in 2004, and the Ph.D. degree in telecommunications from the UPV in 2009. He is a Senior Researcher (Ramon Cajal Fellow) with the Institute of Telecommunications and Multimedia Applications, UPV, where he leads a research group working on next generation broadcasting technologies. He is currently a Research Scholar with the New Jersey Institute of Technology, Newark, NJ, USA. Previously, he hold visiting research appointments at Ericsson Eurolab, Germany, the Royal Institute of Technology, Sweden, the University of Turku, Finland, the Technical University of Braunschweig, Germany and the Fraunhofer Heinrich Hertz Institute, Germany. Since 2008, he has been actively participating in the European Digital Television Standardization Forum DVB in different topics such as upper layer forward error correction, DVB-T2, T2-Lite, and DVB-NGH. In 2013, he joined the U.S. Digital Television Standardization Forum ATSC to work on ATSC 3.0, where he is the Vice-Chairman of the Modulation and Coding Ad-Hoc Group. He is the Editor of the book entitled Next Generation Mobile Broadcasting (CRC Press).

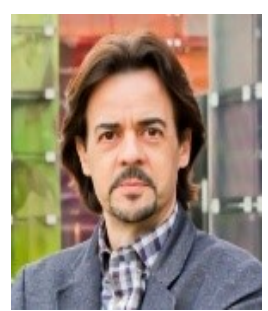

Prof. Narcís Cardona received the M.Sc. degree in Communications Engineering from the ETSI Telecommunications at the Polytechnic University of Catalunya in 1990, and the Ph.D. in Telecommunications from the Polytechnic University of Valencia in 1995. Since October 1990, he is with the Communications Department of the Polytechnic University of Valencia (UPVLC). Prof Cardona is in head of the Mobile Communications Group at the UPVLC, with 30 researchers including assistant professors \& research fellows. Additionally he is the Director of the Mobile Communications Master Degree (since 2006) and Vice-Director of the Research Institute of Telecommunications and Multimedia Applications since 2004. Prof Cardona has led and participated to National research projects and to European projects, Networks of Excellence and other research forums, always in Mobile Communications aspects. At European scale, he has been Vice-Chairman of COST273 Action, Chair of the WG3 of COST2100 in the area of Radio Access Networks, and he is currently the Chairman of the EU Action COST IC1004 since May 2011. From his research work, Prof Cardona has authored eight patents, several books and above 160 research papers. His current areas of interest include mobile channel characterization; planning and optimization tools for cellular systems, RRM techniques applied to personal communications and broadcast cellular hybrid networks. 